Relations industrielles

Industrial Relations

\title{
Laporte, Pierre, Le traité du recours à l'encontre d'un congédiement sans cause juste et suffisante
}

\section{Diane L. Demers}

Volume 48, numéro 2, 1993

URI : https://id.erudit.org/iderudit/050863ar

DOI : https://doi.org/10.7202/050863ar

Aller au sommaire du numéro

Éditeur(s)

Département des relations industrielles de l'Université Laval

ISSN

0034-379X (imprimé)

1703-8138 (numérique)

Découvrir la revue

Citer ce compte rendu

Demers, D. L. (1993). Compte rendu de [Laporte, Pierre, Le traité du recours à

l'encontre d'un congédiement sans cause juste et suffisante]. Relations

industrielles / Industrial Relations, 48(2), 369-372.

https://doi.org/10.7202/050863ar

Tous droits réservés @ Département des relations industrielles de l'Université Laval, 1993
Ce document est protégé par la loi sur le droit d'auteur. L'utilisation des services d'Érudit (y compris la reproduction) est assujettie à sa politique d'utilisation que vous pouvez consulter en ligne.

https://apropos.erudit.org/fr/usagers/politique-dutilisation/ 
The author's treatment of the management of labour within the environmental context of the industrial enterprise operating within its product and labour market, using its specific technology in its production processes provides the reader with an analysis of an industrial relations system as defined by John Dunlop in his classic text. While the author does not deal with the distribution of power in the larger society, he does provide a fairly comprehensive treatment of Industrial Relations as a system operating within the other two environmental contexts.

Taking his analysis beyond the Dunlop model, the author broadens his definition of industrial relations to cover: Work Relations, Employment Relations, and Industrial Relations. This approach provides the reader with an historical perspective on what has been defined by Michael Beer and his associates from the Harvard Busines School as Human Resource Management. The four major HMR policy areas include: Employee Influence (lndustrial Relations), Human Resource Flow, Reward Systems (Employment Relations), and Work Systems (Work Relations) as defined by the Harvard group. The author also maintains a strong linkage between these HRM activities and the economic performance of the firm, based on the concepts of externalization or internalization of these activities.

The purpose of this book is to examine Labour Management from an historical perspective dealing with it in the larger context of the management of the firm and its markets. This approach to the subject requires extensive historical analysis and an examination of corporate histories as well. The book is extremely well organized and presented, providing a detailed historical narrative on the three major topics of investigation. Propositions are set out in the first chapters, analyzed through the narrative and then answered in the concluding chapter. The chapters are well researched, providing a comprehensive account of the topic for the historic period under discussion. Theses are carried through the text providing a long-term historical perspective on them. Each topic is placed within its social, economic and political context.

The author's purpose has been successfully achieved. The value of the book is that it places Labour Management in the broad context of business history and identifies its contribution to the performance of British industry. It provides a well balanced argument, dealing with a complex set of variables over an extended historical period.

The book is a scholarly text, well suited for students of: Management, Industrial Relations, Human Resource Management, Labour Studies and History.

Terry Hercus

University of Manitoba

Le traité du recours à l'encontre d'un congédiement sans cause juste et suffisante, par Pierre LAPORTE, Montréal, Edition Wilson \& Lafleur, 1992, 576 p., ISBN 2-89127-203-X

Cette nouvelle édition du traité s'inscrit dans ce qui, de toute évidence, constitue un point d'intérêt majeur pour le professeur Pierre Laporte; il indique que cet intérêt tient au fait que ce recours « marque une rupture avec la philosophie établie en matière 
de contrat individuel de travail ». Cette rupture résulte de l'attribution au « tribunal d'arbitrage " du pouvoir de sanctionner la décision de l'employeur de mettre fin au contrat individuel de travail d'un employé en ordonnant la réintégration de celui-ci. Pour l'auteur, il s'agit là de la caractéristique centrale du recours qui a conduit à la reconnaissance de la juridiction des tribunaux d'arbitrage sur les contrats individuels de travail. Bien qu'il glisse en quelque sorte sur ce sujet, on peut penser que l'un des objectifs de ce traité était de faire le point sur l'utilisation de ce pouvoir par les tribunaux d'arbitrage vu les conclusions de l'auteur à la toute fin de l'ouvrage.

De fait, il propose dans son traité une analyse-synthèse de la totalité des décisions rendues en vertu du recours de l'article 124 de la Loi sur les normes du travail (L.N.T.) entre le 16 avril 1980 et le $1^{\text {ex }}$ septembre 1991. Le recueil est divisé en deux grandes parties, la première constituant l'analyse-synthèse alors que la seconde présente, sous forme d'annexes, des tableaux-statistiques et un outil de référence, c'est-à-dire quelque 400 décisions, sous forme de fiches, colligées selon trente-cinq thèmes reliés aux motifs de congédiement. De façon assez étonnante, la conclusion, qui se rattache à la première partie, a été placée à la toute fin du recueil après les annexes et la bibliographie.

La première partie se divise en quatre grands chapitres qui visent à situer les personnes, le recours et les décideurs dans le cadre de la loi. Elle inclut les modifications du projet de loi (L.Q. 1990, c. 73) et, à cet égard, quelques remarques s'imposent. L'auteur et les éditeurs ont choisi de mettre en grisé les aspects qui sont de droit nouveau ou qui ont subi des modifications. Cette technique apparaît fort utile dans le premier chapitre qui porte sur les conditions d'admissibilité au recours, où on l'utilise à la fois dans des encadrés et dans de courts passages de texte; elle permet au lecteur de saisir rapidement les changements apportés à la loi et l'utilité ou la pertinence de la jurisprudence antérieure. Par contre, dans le deuxième chapitre qui porte sur la procédure entre la plainte et l'arbitrage, le choix du grisé est tout simplement confondant. On a choisi de mettre tout le texte d'analyse en grisé et d'ajouter un addendum, qui lui n'est pas en grisé, exposant le droit nouveau. Cette méthode qui diffère complètement du chapitre un et s'ajoute au choix de l'auteur d'utiliser l'expression « tribunal d'arbitrage » pour désigner l'instance juridictionnelle passée et actuelle en la matière, rend difficile la tâche du lecteur.

Le premier chapitre vise à rappeler les conditions d'admissibilité du travailleur au recours. Le traitement des aspects généraux de la loi est succinct et clair et la situation contextuelle du recours dans le cadre de la loi est bien présentée. Dans ce chapitre, deux points ont attiré notre attention. Le premier concerne l'analyse des décisions se rapportant au rôle supplétif du recours; elle aurait mérité d'être davantage élaborée car l'exposé de la jurisprudence et de la doctrine rend compte d'un état de confusion persistant sur cette question alors que l'auteur tire des conclusions qui ne s'imposent pas d'ellesmêmes. Le deuxième point porte sur la jurisprudence découlant de la notion de service continu et de l'application de l'article 97 (cession d'entreprise). La présentation des tendances (nettement opposées) antérieures aux décisions de la Cour d'appel présente certes un intérêt, mais on peut s'interroger sur la longueur du développement de cette partie compte tenu que l'auteur lui-même indique que les décisions de la Cour d'appel ont mis fin à la controverse. 
Dans le deuxième chapitre, l'auteur explique les pouvoirs et devoirs du « tribunal d'arbitrage "; il discute des sources et tire de la jurisprudence l'interprétation qui, selon lui, doit en être donnée. Ce qui a retenu particulièrement notre attention est cette affirmation à l'effet que « l'autonomie de l'arbitre en matière de procédure et de preuve lui permettra, s'il le désire, d'intervenir auprès des parties de façon à provoquer un règlement à l'amiable, ce pouvoir d'intervention dans le débat lui était d'ailleurs attribué, la loi l'autorisant à poser à un témoin les questions qu'il croit utiles » (p. 129 ; cette interprétation est reprise à la p. 142). Il ne cite au soutien de cette affirmation qu'une seule décision, ce qui ne nous paraît pas très étonnant compte tenu qu'il s'agit là d'une interprétation pour le moins déconcertante des textes de l'article 100.12 du Code du travail (c.t.). Cette position semble ignorer l'objet même de la procédure d'arbitrage qui est de décider du litige existant entre les parties, procédure qui intervient après les tentatives de règlement confiées à une autre instance.

Les chapitres trois et quatre portent sur les pouvoirs du tribunal d'arbitrage dans l'évaluation de la plainte et de la réparation qui en découle. L'auteur propose dans le chapitre trois une analyse de la jurisprudence relative à la portée du terme congédiement. A cet égard, il passe en revue l'ensemble des pratiques en matière de rupture du lien d'emploi et regarde la position tant des tribunaux d'arbitrage que des tribunaux supérieurs sur la question. Il apparaît évident que l'exercice a été fait de façon attentive et méticuleuse. Nous croyons cependant qu'une lecture prudente s'impose lorsque l'auteur propose de mettre en opposition ce qui relève de paramètres différents dans l'identification des tendances jurisprudentielles. Il en est ainsi notamment lorsqu'il discute de décisions relatives au service continu face aux mises à pied pour motifs économiques; il cite, à titre d'exemple d'une tendance, une décision de R. Blouin fondée sur la question de reconnaissance du service continu comme critère non discriminatoire de sélection du salarié mis à pied, l'employeur avant décidé de garder à son service le plus jeune, alors que l'exemple utilisé pour illustrer la tendance opposée relève du fait que la décision de l'employeur repose sur une sélection fondée sur l'état de mésentente entre celui-ci et l'employé (p. 180-182).

Le quatrième et dernier chapitre traite des pouvoirs de réparation du tribunal d'arbitrage. On y trouve dans une première partie un rappel de la situation constitutionnelle du tribunal à l'égard de ce pouvoir de réparation. Par la suite, l'auteur discute de la portée et des limites des trois énoncés de l'article 128 L.N.T. Relativement au pouvoir de réintégration, il présente une forme de bilan des motifs invoqués ou retenus par les arbitres pour réintégrer ou non le salarié puis il passe rapidement sur le paiement du salaire perdu. Dans la dernière section de cette partie, l'auteur présente en huit sous-sections les diverses formes qu'a pris le pouvoir de « rendre toute autre décision » prévu à l'article 128 (3) de la loi; il discute notamment des dommages-intérêts (matériels) et des dommages moraux. L'auteur mentionne que dans le premier cas, la jurisprudence est claire : les dommages qui résultent de la rupture injustifiée du contrat relèvent de la juridiction du tribunal d'arbitrage. D'autre part, il propose à l'égard des dommages moraux qu'avant 1989 les assises d'une telle décision étaient fragiles mais que depuis les arrêts Ste-Anne Nackawick et Fosy c. Bell, le tribunal d'arbitrage a effectivement juridiction. Il énonce cette conclusion en se fondant sur la prétention que le « tribunal d'arbitrage» est, par assimilation, un arbitre de grief au sens du Code du travail. Nous croyons 
cependant que l'arrêt de la Cour suprême ne peut pas trouver application par simple analogie. Il faudra que le dommage résulte de la rupture du contrat prévue à l'article 124, puisque c'est dans la mesure où le droit a pris naissance dans la convention collective que la Cour a reconnu le pouvoir à l'arbitre de grief.

L'auteur termine ce chapitre par le constat qu'à ce jour moins de la moitié des salariés ayant été l'objet d'un congédiement sans cause juste et suffisante ont été réintégrés. Cependant il propose dans sa conclusion de l'ouvrage un tempérament aux déductions que l'on pourrait tirer de ces informations en soulignant qu'il faut lire ces données en perspective avec le nombre de plaintes déposées auprès de la Commission des normes du travail et en tenant compte de l'effet dissuasif que l'existence d'un tel recours comporte. Bien qu'il ne s'agisse pas de l'objectif premier de ce traité, cette conclusion de l'auteur est intéressante. Quant à l'analyse-synthèse du contentieux entourant le recours de l'article 124 L.N.T., nous croyons que le professeur Laporte a réussi à exposer l'état du droit sur cette question de façon claire et compréhensive, ce qui fait de ce traité un outil de référence utile pour l'ensemble des pratriciens et praticiennes.

Diane L. DEMERS

Montréal

Les normes internationales du travail, Manuel d'éducation ouvrière, troisième édition (révisée), Genève, Bureau international du travail, 1992, 143 p., ISBN 92-2-206445-3

Manuel d'éducation ouvrière, cet ouvrage présente l'activité normative de l'Organisation internationale du travail. Après avoir retracé les origines de l'institution savait-on qu'au milieu du siècle dernier un industriel français du nom de LeGrand avait fait campagne en vue de faire adopter certaines normes du travail européennes? -, il en décrit la structure tripartite et les organes, de même que le mode d'élaboration et d'adoption des conventions et recommandations.

Suit une synthèse de l'objet et du contenu de ces instruments : à la fin de 1990 , on comptait cent soixante et onze conventions et cent soixante-dix-huit recommandations traitant notamment de la liberté syndicale, de la liberté du travail, de l'égalité de chances et de traitement entre la main-d'œuvre masculine et la main-d'œuvre féminine, de l'emploi, de la formation professionnelle et du placement, de l'inspection et des statistiques du travail, de la négociation collective, du tripartisme, du salaire et autres conditions de travail, de la santé et de la sécurité du travail, de la cessation de la relation de travail, de même que d'aspects majeurs de la sécurité sociale - soins médicaux, indemnité de maladie, prestations de maternité, d'invalidité, de vieillesse, de chômage et prestations en cas d'accidents du travail et de maladies professionnelles.

Une seconde moitié, plus ou moins, du texte expose les modes d'application de ces normes : soumission initiale aux autorités gouvernementales compétentes, pour ratification éventuelle, ou, à tout le moins, en vue de la confrontation périodique de l'état de la législation interne et de son contenu : procédure normale de contrôle, de l'accomplissement des obligations des États membres - examen des rapports gouvernementaux 